\title{
Gene Expression Profiling of Placentas Affected by Pre-Eclampsia
}

\author{
Anne Mette Hoegh, ${ }^{1}$ Rehannah Borup, ${ }^{2}$ Finn Cilius Nielsen, ${ }^{2}$ Steen Sørensen, ${ }^{1}$ \\ and Thomas V. F. Hviid ${ }^{2}$ \\ ${ }^{1}$ Department of Clinical Biochemistry, Hvidovre Hospital, University of Copenhagen, Kettegaard Allé 30, 2650 Hvidovre, Denmark \\ ${ }^{2}$ Department of Clinical Biochemistry, Rigshospitalet, University of Copenhagen, Blegdamsvej 9, 2100 Copenhagen, Denmark
}

Correspondence should be addressed to Steen Sørensen, steen.soerensen@hvh.regionh.dk

Received 28 July 2009; Revised 29 October 2009; Accepted 24 November 2009

Academic Editor: Wenjiang J. Fu

Copyright (C) 2010 Anne Mette Hoegh et al. This is an open access article distributed under the Creative Commons Attribution License, which permits unrestricted use, distribution, and reproduction in any medium, provided the original work is properly cited.

\begin{abstract}
Several studies point to the placenta as the primary cause of pre-eclampsia. Our objective was to identify placental genes that may contribute to the development of pre-eclampsia. RNA was purified from tissue biopsies from eleven pre-eclamptic placentas and eighteen normal controls. Messenger RNA expression from pooled samples was analysed by microarrays. Verification of the expression of selected genes was performed using real-time PCR. A surprisingly low number of genes $(21$ out of 15,000$)$ were identified as differentially expressed. Among these were genes not previously associated with pre-eclampsia as bradykinin B1 receptor and a 14-3-3 protein, but also genes that have already been connected with pre-eclampsia, for example, inhibin beta A subunit and leptin. A low number of genes were repeatedly identified as differentially expressed, because they may represent the endpoint of a cascade of events effectuated throughout gestation. They were associated with transcriptional regulation and vasoregulative pathways, along with a number of hypothetical proteins and gene sequences with unknown functions.
\end{abstract}

\section{Introduction}

One of the leading causes of mortality and morbidity amongst pregnant women and their offspring is preeclampsia (PE), affecting $2 \%-8 \%$ of all pregnancies depending on the criteria of diagnosis and ethnic population [1]. Pre-eclampsia is defined by hypertension and proteinuria in the pregnant woman. The symptoms manifest themselves in the late second or third trimester of pregnancy but there is most likely a long subclinical course of events before the debut of symptoms [2]. A rare complication of the condition is eclampsia involving encephalopathia and seizures, sometimes with a fatal result. For the fetus, pre-eclampsia commonly leads to intrauterine growth retardation (IUGR), low birth weight, prematurity, or perinatal death [3]. In early pregnancy cytotrophoblast cells invade the placental bed, resulting in a physiological remodelling of the spiral arteries. During the normal non-pre-eclamptic invasion process, the trophoblast becomes embedded in the vessel wall and the muscular and elastic components are disrupted, transforming the arteries into dilated low resistance vessels. These vessels are unable to constrict in response to vasoactive stimuli thereby ensuring an adequate blood supply to the placenta and the fetus. In pre-eclampsia, the trophoblastic invasion is impaired and the spiral arteries retain their endothelial lining and musculature, compromising blood flow into the intervillous space and thereby restricting the blood supply to the placenta and the fetus giving rise to ischemic hypoxia and oxidative stress [4-6].

The cause of pre-eclampsia is unknown, but it is linked to the presence of a placenta, since pre-eclampsia can occur in molar pregnancies where a placenta, but no fetus, is present [7]. Also, the only effective treatment of pre-eclampsia is delivery of the placenta. Studies have shown that some families are predisposed for development of pre-eclampsia and it has therefore been concluded that a hereditary element exists $[8,9]$, most likely to be the result of an interaction between several different genes.

In an attempt to identify genes that may be of importance for the pathological and pathophysiological changes seen in the pre-eclamptic placentas and to identify pre-eclamptic factors, which may be released into the maternal blood circulation, we investigated the gene expression in term placentas from uncomplicated pregnancies and pregnancies 
affected by pre-eclampsia and which may be involved in the endothelial dysfunction. Throughout this paper, we use the phrase "term" to designate the end of pregnancy by delivery at any time during gestation (rather than just full term) unless otherwise specified. All the placentas investigated are from the third trimester. It must therefore be remembered that the pathways which appear to be regulated in this study represent the endpoint of what must be presumed to be a cascade of events effectuated throughout gestation.

\section{Materials and Methods}

2.1. Study Population. This is a case-control-study consisting of pregnant women with pre-eclampsia and a control group with an uncomplicated pregnancy. The sole inclusion criteria used for the women suffering pre-eclampsia were a blood pressure $\geq 140 / 90 \mathrm{mmHg}$ or a rise in diastolic blood pressure of $\geq 30 \mathrm{mmHg}$, proteinuria of $\geq 300 \mathrm{mg} / 24$ hours or $\geq 300 \mathrm{mg} / \mathrm{L}$ or $\geq 1+$ by urinary dipstick analysis. Personal or family history of PE was not recorded. All were self-reported nonsmokers. The project was in accordance with the Helsinki II declaration and approved by the local Ethics Committee. The women gave their informed consent prior to sampling. An overview of the clinical data of the included pregnant women is given in Table 1. The method of delivery differs between the cases and controls. Most cases relate to vaginal deliveries and most controls relate to elective Caesarean sections. This may have influenced the comparison of genes implicated in the induction and progression of labour since these genes may be differentially expressed due to the process of labour rather than the pathogenesis of PE. A number of additional control subjects that were not included in the array analysis were also analyzed by real-time PCR. The majority of these controls were multiparous women in whom, theoretically, a different placental gene expression may exist compared with that of primiparous women.

2.2. Tissue Samples. Placental biopsies were obtained from the maternal side of term placentas at a maximum of 30 minutes after delivery. Biopsies of approximately $0.2-0.5 \mathrm{~cm}^{3}$ were taken from the center of the cotyledons evenly across the placenta. Some biopsies were transferred to 10 volumes of RNAlater solution (Ambion, Austin, Texas) and incubated at $4^{\circ} \mathrm{C}$ over night prior to freezing at $-80^{\circ} \mathrm{C}$. Others were immediately processed for RNA purification by lysis in Trizol (Invitrogen, Paisley, UK), followed by homogenisation and centrifugation to remove cellular debris. Hereafter, the lysate was frozen at $-80^{\circ} \mathrm{C}$. From a subset of the placentas, biopsies were snap-frozen in liquid nitrogen and stored at $-80^{\circ} \mathrm{C}$.

2.3. Purification of RNA from the Tissue Samples. Before analysis, the RNA was pooled into a total of six groups; three groups each consisting of equal amounts of RNA from three pre-eclamptic placentas and three groups each consisting of equal amounts of RNA from three matched normal controls (i.e., triplicate experiments a, b, and c with paired cases and controls, see Table 1). The placental samples were matched for maternal age, parity, gestational week at delivery, smoking habits, the gender of the child, and when possible, method of delivery (but as Table 1 shows this was not possible in all cases).

RNA was purified from two biopsies (each of $30 \mathrm{mg}$ cut from the original larger biopsies) from each placenta using Trizol (Invitrogen) according to the instructions of the manufacturer. The RNA preparation was subjected to a clean-up procedure using RNeasy Mini Kit (QIAGEN, $\mathrm{GmbH}$, Hilden, Germany) according to the instructions from the manufacturer. RNA concentrations were measured by spectrophotometer.

2.4. Gene Expression Analysis. The gene expression of the samples was analysed using GeneChip (HG-U133A, Affymetrix Inc., Santa Clara, CA, USA) microarrays according to the instructions of the manufacturer. In short, mRNA was reverse transcribed into first strand cDNA using a T7-Oligo(dT) Promoter primer (GenSet/Proligo, Paris, France), followed by a RNaseH-mediated secondstrand cDNA synthesis. The double-stranded cDNA was purified and used as template in an in vitro transcription (IVT) reaction in the presence of biotin-labelled UTP and CTP (Enzo Diagnostics) to yield amplified and biotinlabelled cRNA (ENZO BioArray HighYield RNA Transcript Labelling Kit; Affymetrix Inc.). Biotin-labelled cRNA was cleaned, extracted, and fragmented randomly to approximately $250 \mathrm{bp}$ and hybridized for 16 hours to an Affymetrix HG-U133A array, containing approximately 22,000 probe sets representing approximately 15,000 well substantiated genes. The arrays were washed, stained with phycoerytrin streptavidin (SAPE) using the Affymetrix Fluidics Station 400 and fluorescent image files were produced using the Affymetrix GeneArray scanner 2500.

Evaluation of the results was performed using the DNAChip Analyzer (dChip) programme, a freeware programme available at http://biosun1.harvard.edu/complab/dchip [10]. The image files (cel files) were imported into the software, and the array files were normalized using the multiarray invariant-set normalization method. All arrays in the experiment were normalized to the array with the median overall brightness, which was selected as the baseline. The invariantset normalization is based on probe values belonging to nondifferentially expressed genes between the array being normalized and the baseline array (the invariant set). The invariant set is used to generate a piecewise linear median curve between the baseline probes and the experiment probes, which is used at the normalization curve.

$M$ versus A (MVA) plots were constructed after sample normalization using the Affy package from the R, BioConductor (http://www.bioconductor.org) to check the correlation between the replicate samples in each group and the variation between the normal control group and PE group, respectively. In the MVA plots the $A$-axis plots the average $\log$ intensity $(\log$ chip $1+\log$ chip2)/2 and the $M$ axis plots the log of ratio (chip1/chip2). A matrix of MVA plots was constructed for Control, Pre-eclampsia, and both sample groups, where the MVA plots are showed in the upper triangle and the IQR (interquartile range) of the Ms and 
TABLE 1: Clinical data on the included women and deliveries.

\begin{tabular}{|c|c|c|c|c|c|c|c|c|c|c|c|c|c|c|}
\hline \multirow[b]{2}{*}{$\begin{array}{l}\text { Chip } \\
\text { pool }\end{array}$} & \multicolumn{7}{|c|}{ Pre-eclampsia } & \multicolumn{7}{|c|}{ Control } \\
\hline & Age & Para & $\begin{array}{l}\text { Weeks of } \\
\text { gestation }\end{array}$ & $\begin{array}{l}\text { Sex of } \\
\text { child }\end{array}$ & Smoking & Delivery & Co-morbidities & $\begin{array}{l}\text { Chip } \\
\text { pool }\end{array}$ & Age & Para & $\begin{array}{l}\text { Weeks of } \\
\text { gestation }\end{array}$ & $\begin{array}{l}\text { Sex of } \\
\text { child }\end{array}$ & Smoking & Delivery \\
\hline A & 26 & I & 39 & M & $\mathrm{N}$ & IL & & $A$ & 27 & $I$ & 39 & $M$ & $N$ & $E S$ \\
\hline$A$ & 29 & $I$ & 37 & $F$ & $N$ & $I L$ & & $A$ & 35 & $I$ & 38 & $F$ & $N$ & $E S$ \\
\hline$A$ & 29 & $I$ & 39 & $M$ & $N$ & $I L$ & & $A$ & 35 & $I$ & 38 & $M$ & $N$ & $E S$ \\
\hline B & 32 & I & 33 & M & $\mathrm{N}$ & ES & & $B$ & 33 & $I$ & 35 & $M$ & $N$ & $I L+S$ \\
\hline B & 25 & I & 39 & $\mathrm{~F}$ & $\mathrm{~N}$ & IL & $*$ & B & 26 & I & 39 & $\mathrm{~F}$ & $\mathrm{~N}$ & ES \\
\hline B & 26 & I & 40 & M & $\mathrm{N}$ & IL & & $B$ & 28 & $I$ & 39 & $M$ & $N$ & $E S$ \\
\hline C & 24 & I & 38 & $\mathrm{~F}$ & $\mathrm{~N}$ & V unc & & $\mathrm{C}$ & 32 & I & 39 & $\mathrm{~F}$ & $\mathrm{~N}$ & V unc \\
\hline C & 29 & II & 37 & $\mathrm{~F}$ & $\mathrm{~N}$ & V unc & & $\mathrm{C}$ & 30 & II & 39 & $\mathrm{~F}$ & $\mathrm{~N}$ & ES \\
\hline C & 35 & I & 37 & $\mathrm{M}$ & $\mathrm{N}$ & V unc & & C & 33 & I & 38 & M & $\mathrm{N}$ & ES \\
\hline- & 29 & I & 32 & $\mathrm{M}$ & $\mathrm{N}$ & AS & $* *$ & & & & & & & \\
\hline \multirow[t]{10}{*}{ - } & 24 & I & 36 & M & $\mathrm{N}$ & AS & & & & & & & & \\
\hline & & & & & & & & - & 27 & II & 38 & M & $\mathrm{N}$ & ES \\
\hline & & & & & & & & - & 34 & $\mathrm{~V}$ & 38 & M & $\mathrm{N}$ & ES \\
\hline & & & & & & & & - & 29 & II & 39 & M & $\mathrm{N}$ & ES \\
\hline & & & & & & & & - & 38 & IV & 38 & $\mathrm{~F}$ & $\mathrm{~N}$ & ES \\
\hline & & & & & & & & - & 36 & III & 37 & M & $\mathrm{N}$ & ES \\
\hline & & & & & & & & - & 25 & $\mathrm{I}$ & 39 & $\mathrm{~F}$ & $\mathrm{~N}$ & ES \\
\hline & & & & & & & & - & 35 & II & 38 & M & $\mathrm{N}$ & ES \\
\hline & & & & & & & & - & 31 & II & 38 & M & $\mathrm{N}$ & ES \\
\hline & & & & & & & & - & 33 & II & 38 & $\mathrm{~F}$ & $\mathrm{~N}$ & AS \\
\hline
\end{tabular}

Italics mean no real-time PCR experiments performed. Paired pre-eclamptics and controls for microarray analysis are listed on the same line in the table. IL $=$ induction of labour, ES = elective sectio, AS = acute section, $\mathrm{V}$ unc = uncomplicated vaginal delivery Co-morbidities: ${ }^{*}$ abortus imminens, ${ }^{* *}$ possible HELLP.

the Median of the As are displayed in the lower triangle. A model-based expression index was calculated using perfect match signals only and comparisons between sample groups were performed. For all comparison analyses, the following parameters were used: Minimum fold change of 1.2 fold, minimum absolute change in signal intensity of 50 units and a $P$-value for the change in signal intensity of.05 or less. The dChip software calculates the upper and lower bounds of a 90 percent confidence interval for the fold change. We have filtered our results by the lower bound fold change, which is the more conservative estimate of the fold change. The triplicate pairs of samples were analyzed on six separate arrays. For analysis, the control arrays were grouped together and their mean signals were compared with the mean signals of the case arrays. From the resulting list of genes generated by dChip, we chose a number of genes to verify by real-time PCR.

2.5. Verification of Results from the Gene Expression Analysis by Real-Time PCR. A number of genes found to be differentially expressed between case and control placentas were examined by real-time PCR using a LightCycler instrument (Roche). The genes we focused on were fibulin 1A, inhibin, leptin, and tyrosine 3-monooxygenase/tryptophan 5-monooxygenase activation protein. Templates for the verification were the RNA preparations from individual placentas. Nine RNA preparations originated from preeclamptic placentas and twelve preparations originated from control placentas. A minimum of two different biopsies for each placenta were included in the preparation. From the RNA preparations, cDNA was synthesized using Superscript II RNase H- Reverse Transcriptase (Invitrogen, CA, USA) according to the instructions of the manufacturer. Equal amounts of total RNA $(1.7 \mu \mathrm{g})$ were used for all reverse transcription reactions. All cDNA templates were diluted $25 \times$ in water before analysis. All primers were used in a final concentration of $0.5 \mu \mathrm{M}$ each and the $\mathrm{MgCl}_{2}$ concentration was $2 \mathrm{mM}$ for all reactions. Primers and PCR conditions for each gene are listed in Table 2. A varying number of touchdown cycles were performed before the PCR to improve specificity. The annealing temperature and the number of cycles for which it was used are listed in Table 2. All reactions were carried out in a total volume of $16 \mu \mathrm{L}$, using $1.6 \mu \mathrm{L}$ of diluted template and $1.6 \mu \mathrm{L}$ LightCycler FastStart Reaction Mix SYBR Green I (Roche) prepared according to the manufacturer's instructions. Standard curves were performed for each run to ensure the comparability of the samples. For generation of a standard curve of sample fluorescence against relative template concentration, we used a pool of equal amounts of all 22 individual cDNA preparations as template. Undiluted template pool and dilutions of $25 \times, 50 \times$, and $100 \times$ were used to generate the standard curve. All the individual results were evaluated against this curve. Furthermore, GAPDH expression was determined for all samples and used for normalization before statistical analysis was carried out. 
TABLE 2: Primers and PCR conditions.

\begin{tabular}{|c|c|c|c|c|c|c|c|}
\hline Gene target & Forward primer $5^{\prime}-3^{\prime}$ & Reverse primer $5^{\prime}-3^{\prime}$ & $\begin{array}{c}\mathrm{TD}^{\mathrm{a}} \mathrm{a} \\
{ }^{\circ} \mathrm{C} / \mathrm{c}\end{array}$ & $\begin{array}{c}\mathrm{TD}^{\mathrm{a}} \mathrm{b} \\
{ }^{\circ} \mathrm{C} / \mathrm{c}\end{array}$ & $\begin{array}{l}\mathrm{TD}^{\mathrm{a}} \mathrm{c} \\
{ }^{\circ} \mathrm{C} / \mathrm{c}\end{array}$ & $\begin{array}{c}\mathrm{TD}^{\mathrm{a}} \mathrm{d} \\
{ }^{\circ} \mathrm{C} / \mathrm{c}\end{array}$ & $\begin{array}{c}\mathrm{PCR}^{\mathrm{b}} \\
{ }^{\circ} \mathrm{C} / \mathrm{c}\end{array}$ \\
\hline Fibulin 1A & aagttggcaggagtggagac & cccccataggtgaatcacag & $58 / 2$ & $57 / 2$ & $56 / 2$ & - & $55 / 35$ \\
\hline sFLT-3 & taagcacaccacgcccagtc & aagaccgcttgccagctacg & $69 / 2$ & $68 / 2$ & $67 / 2$ & - & $66 / 30$ \\
\hline Inhibin & gcttcatgtgggcaaagtcg & ccccctttaagcccacttcc & $64 / 2$ & $62 /$ & $60 /$ & $59 / 2$ & $58 / 30$ \\
\hline Leptin & gtccaagctgtgcccatcc & cccaggctgtccaaggtctc & $64 / 2$ & $62 /$ & $60 /$ & $59 / 2$ & $58 / 30$ \\
\hline
\end{tabular}

${ }^{(a)}$ Touchdown cycle, annealing temperature/number of cycles.

(b) PCR, annealing temperature/number of cycles.
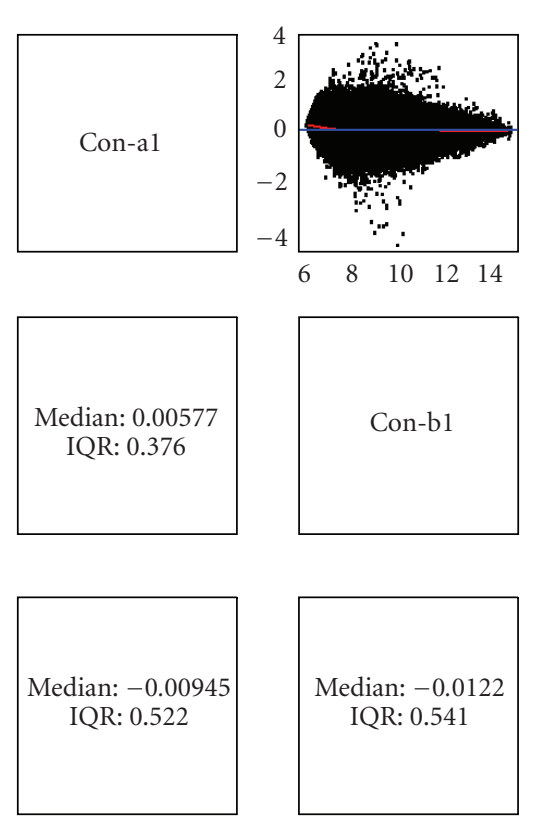

M
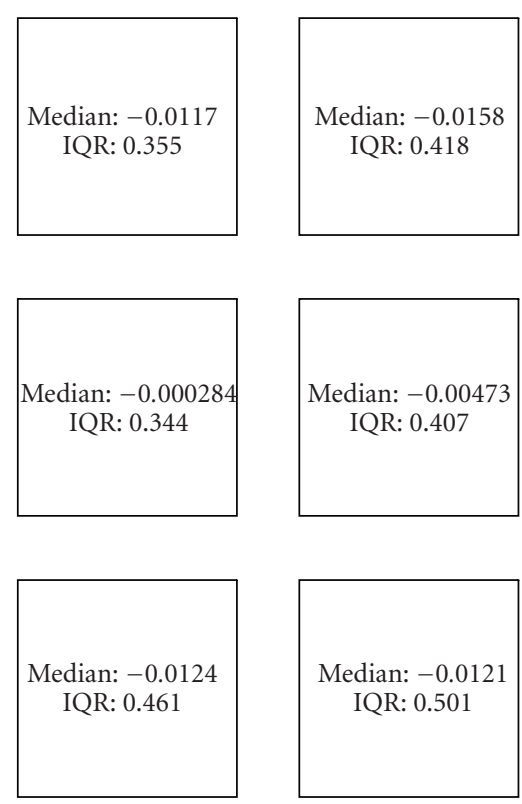

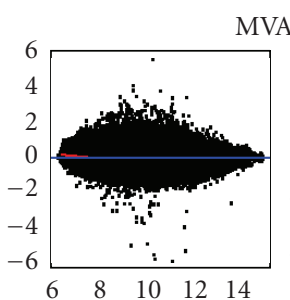

MVA plot
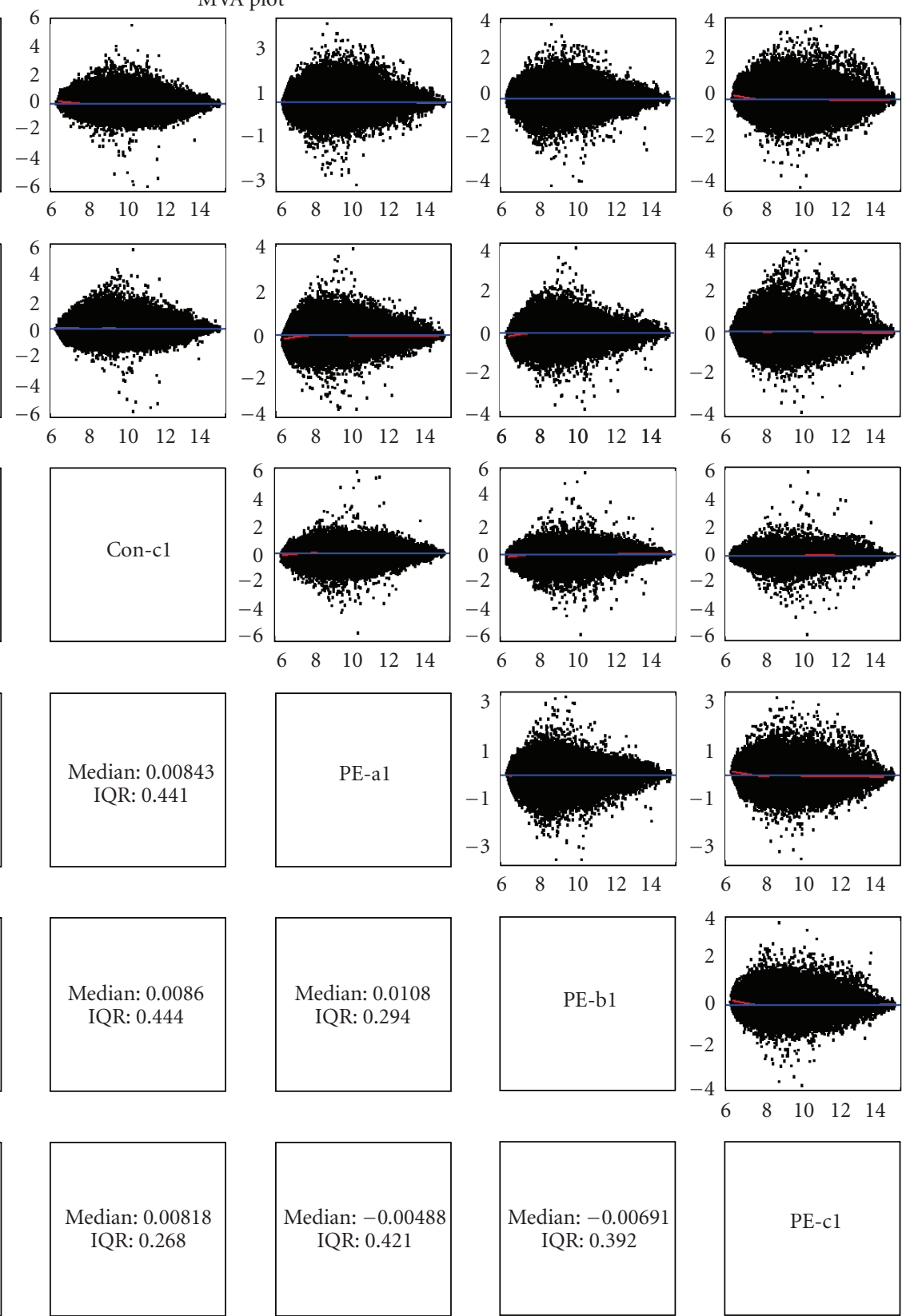

A

FIGURE 1: The interquartile range (IQR) values for the MVA plots show that the variation between the arrays within each group (case versus case or control versus control) is in the same range as the variation between the groups (case versus control). This indicates a high similarity between the case and control groups which is also apparent by the relatively low number of differentially expressed genes identified. 
2.6. Statistical Analysis. The results generated from the realtime PCR experiments were compared using Mann-Whitney test.

\section{Results}

3.1. Microarray Data. A total of 21 genes were found to be differentially expressed between the arrays by dChip analysis, of these 9 were downregulated and 12 upregulated. The genes are listed in Table 3. A matrix of $\mathrm{M}$ versus $\mathrm{A}$ (MVA) plots was constructed for Controls, Pre-eclampsia and both sample groups (Figure 1). The interquartile range (IQR) values for the MVA plots showed that the variation between the arrays within each group (case versus case or control versus control) was in the same range as the variation between the groups (case versus control). This indicated a high similarity between the case and control groups, which is also apparent by the relatively low number of differentially expressed genes identified.

The genes chosen for real-time PCR verification were tyrosine 3-monooxygenase/tryptophan 5-monooxygenase activation protein and fibulin 1A on the basis of their fold change and their novelty in relation to the pathogenesis of PE. Inhibin and leptin have previously been associated with PE and were included as a verification of the PCR setup.

3.2. qRT-PCR Data. The relative expression levels calculated from the real-time PCR experiments are depicted in Figure 2. The regulation of fibulin between cases and controls observed on the arrays was not confirmed. The array analysis result was shown to have been influenced by a single sample expressing large amount of fibulin mRNA and the difference in expression observed by real-time PCR did not reach statistical significance $(P=.619 ; \mathrm{FC} 1.46)$. The differential expression indicated by the array analysis for leptin was verified $(P=.012 ; \mathrm{FC} \mathrm{3.41)}$. The difference in expression levels for inhibin and tyrosine 3-monooxygenase/tryptophan 5monooxygenase activation protein did not reach significance $(P=.500, \mathrm{FC} 1.13 ; P=.166, \mathrm{FC}-1.27)$. The expression patterns for the genes examined by real-time PCR thus in general confirm the trends observed by microarray analysis.

\section{Discussion}

The most interesting feature of this study is the similarity between the arrays and the potential significance of the expression of 21 out of possible 15,000 genes. In hindsight that may not be very surprising. It is generally agreed that pre-eclampsia has a long subclinical course before the onset of symptoms and the main part of the causative placental molecular events that lead to the development of PE is temporary and may not be apparent at term. Also, tissues other than the placenta, such as the maternal vascular endothelium, may play a role in the pathogenesis of PE. This study includes cases of both late $(n=9)$ and early onset PE $(n=2)$ (cut-off 34 weeks of gestation). The pooled samples may mask any differences between the pathogenesis of late and early onset PE, but should on the other hand ensure that any gene expression differences detected is more likely a representative of the disease as a whole. We have also included true biological replicates in the current study design by pooling samples from other completely independent cases and controls; this is in contrast to several other published studies regarding gene expression in pre-eclamptic placentas, and should be a strength of the current study.

In the following, descriptions of the transcripts found to be differentially expressed by microarray analysis and/or further analysis are given and their relevance in relation to the pre-eclamptic syndrome is discussed. The sequences have been grouped in sections according to their possible influence on certain characteristics of pre-eclampsia: inadequate placentation, oxidative stress, inflammatory response, hypertension, and metabolism. Throughout the discussion, one should bear in mind that the results from this study rests on mRNA analysis only and that the mRNA expression profile of any biological system may not necessarily reflect the protein profile. Finally, a brief evaluation of the microarray experiment is given, followed by a general discussion, and a conclusion.

\subsection{Gene Descriptions and Regulations}

4.1.1. Inadequate Placentation. A complex of different thyroid hormone receptor-associated proteins (TRAPs) play a role as coactivators for thyroid hormone dependent transcriptional activation [11]. The placenta may be a thyroid hormone dependent tissue. Stimulation by thyroid hormones of trophoblast endocrine function resulting in enhanced production of human placental lactogen and human chorionic gonadotropin has been reported [12]. It has also been shown that L-triiodothyronine enhances the production of epidermal growth factor, a potent trophoblast mitogen [13]. Thyroid hormones may thus have an important role in villous development and placentation, in part mediated by the interaction between endocrine and autocrine factors [14]. We found a $240 \mathrm{kDa}$ subunit (TRAP 240) expression decreased by 1.32 fold, which may indicate an impaired thyroid hormone signalling perhaps involved in the inadequate placentation observed in PE.

4.1.2. Oxidative Stress. The selenoprotein thioredoxin reductase (TrxR1) is a cytosolic antioxidant enzyme known to reduce many compounds in addition to thioredoxin, its principle protein substrate. It functions to eliminate reactive oxygen species (ROS) and regenerate oxidatively damaged proteins. Some have found that the levels of oxireductases, thioredoxin reductase included, were increased approximately 2- to 3-fold in pre-eclamptic placentas compared to normal placentas and it was speculated that this might be an adaptive response to the oxidative stress observed in PE [15]. The same group of investigators has also shown that oxidative stress does indeed induce thioredoxin reductase expression in the trophoblast [16]. However, we found thioredoxin reductase to be downregulated (1.57 fold) in the pre-eclamptic placentas compared with the normal controls. Failure to increase thioredoxin reductase may further promote a condition of oxidative stress. 
TABLE 3: Regulation of genes in pre-eclamptic placentas compared to controls.

\begin{tabular}{|c|c|c|c|}
\hline Accession $^{1}$ & Gene of interest & Fold change & $P$-value \\
\hline \multicolumn{4}{|c|}{ Antioxidant enzymes } \\
\hline NM_003330.1 & thioredoxin reductase 1 & -1.57 & .025 \\
\hline \multicolumn{4}{|c|}{ Eicosanoid metabolism } \\
\hline U63296.1 & hydroxyprostaglandin dehydrogenase $15-\left(\mathrm{NAD}^{+}\right)$, & 1.48 & .027 \\
\hline BC005939.1 & prostaglandin D2 synthase $21 \mathrm{kDa}$ (brain) & -1.51 & .031 \\
\hline \multicolumn{4}{|c|}{ Energy metabolism } \\
\hline AI761561 & hexokinase 2 & 1.59 & .045 \\
\hline NM_000230.1 & leptin & 2.94 & .025 \\
\hline AA502643 & $\begin{array}{l}\text { tyrosine 3-monooxygenase/tryptophan } \\
\text { 5-monooxygenase activation protein e }\end{array}$ & -1.65 & .005 \\
\hline \multicolumn{4}{|c|}{ Inhibin-activin system } \\
\hline NM_002192.1 & $\begin{array}{l}\text { inhibin, beta A subunit (activin A, activin AB alpha } \\
\text { polypeptide) }\end{array}$ & 2.06 & .007 \\
\hline \multicolumn{4}{|l|}{ Matrix proteins } \\
\hline NM_000494.1 & collagen, type XVII, alpha 1 & 1.50 & .039 \\
\hline NM_006487.1 & fibulin $1 \mathrm{~A}$ & -2.49 & .009 \\
\hline \multicolumn{4}{|c|}{ Transcription factors } \\
\hline AA972711 & zinc finger protein 292 & -1.37 & .024 \\
\hline NM_005121.1 & $\begin{array}{l}\text { thyroid hormone receptor-associated protein, } 240 \mathrm{kDa} \\
\text { subunit }\end{array}$ & -1.32 & .012 \\
\hline \multicolumn{4}{|l|}{ Vascular factors } \\
\hline NM_000710.1 & bradykinin $\mathrm{B} 1$ receptor & -1.44 & .024 \\
\hline NM_013332.1 & hypoxia-inducible protein 2 & 1.61 & .029 \\
\hline \multicolumn{4}{|l|}{ Miscellaneous } \\
\hline AI206718 & ESTs, Weakly similar to zinc finger protein 339 & 1.52 & .024 \\
\hline AF052169.1 & hypothetical protein $\mathrm{BC} 013764$ & -1.46 & .033 \\
\hline AF007149.1 & hypothetical protein LOC257407 & -1.40 & .035 \\
\hline AK025495.1 & KIAA0790 protein & 1.56 & .041 \\
\hline AK027231.1 & KIAA1102 protein & 1.54 & .003 \\
\hline AB033025.1 & KIAA1199 protein & 1.59 & .040 \\
\hline NM_004844.1 & $\begin{array}{l}\text { SH3-domain binding protein } 5 \text { (BTK-associated), } \\
\text { (signal transduction) }\end{array}$ & 1.58 & .030 \\
\hline AF016535.1 & $\begin{array}{l}\text { ATP-binding cassette, sub-family B (MDR/TAP), } \\
\text { member 1, (transporter activity) }\end{array}$ & 1.45 & .037 \\
\hline
\end{tabular}

${ }^{1}$ Genbank accession number.

4.1.3. Inflammatory Response. Tyrosine 3-monooxygenase/ tryptophan 5-monooxygenase activation protein, epsilon variant, is a so-called 14-3-3 protein. Interestingly, 14-33 proteins have also been found to exhibit antiapoptotic characteristics [17]. A decrease of 14-3-3, as identified by microarray analysis and real-time PCR, could therefore increase the occurrence of apoptosis in placental cells and thereby also increase the level of placental cellular debris shed into the maternal blood circulation. Syncytiotrophoblast microvillous membrane (STBM) fragments have been found at higher concentrations in the circulation of pre-eclamptic women compared to women with uncomplicated pregnancies [18]. It has been proposed that STBMs contribute to the vascular endothelial dysfunction of pre-eclamptic women.
4.1.4. Inhibin, Beta A Subunit. Activins and inhibins are growth and differentiation factors belonging to the transforming growth factor-beta superfamily. The activins are dimeric proteins consisting of two inhibin beta subunits (beta A homodimer or beta A beta B heterodimer). Either of the beta subunits in combination with an inhibin alpha subunit yield the protein inhibin, which has functions quite opposite of those of activin in that inhibin will inhibit the actions of activins. It has been shown that women with pre-eclampsia have increased serum levels of inhibin A and activin A [19]. Inhibin mRNA levels in pre-eclamptic placentas are elevated compared to normal controls [20] and activin A mRNA and protein are also increased [21, 22] suggesting that placental expression may contribute 


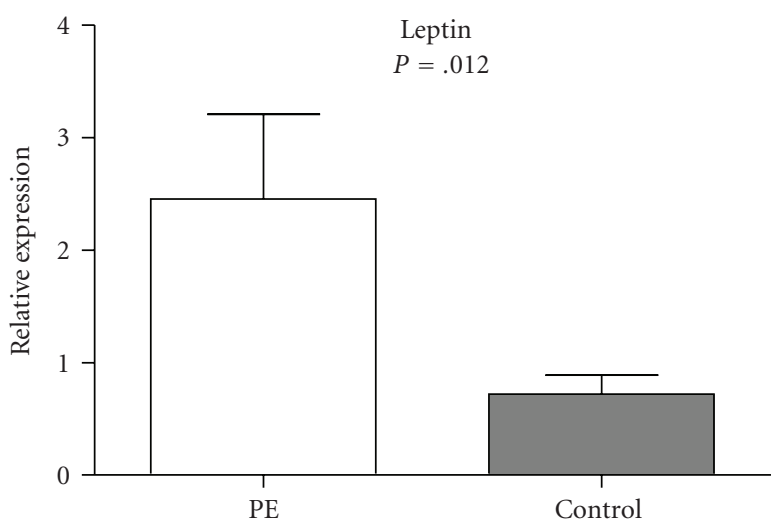

(a)

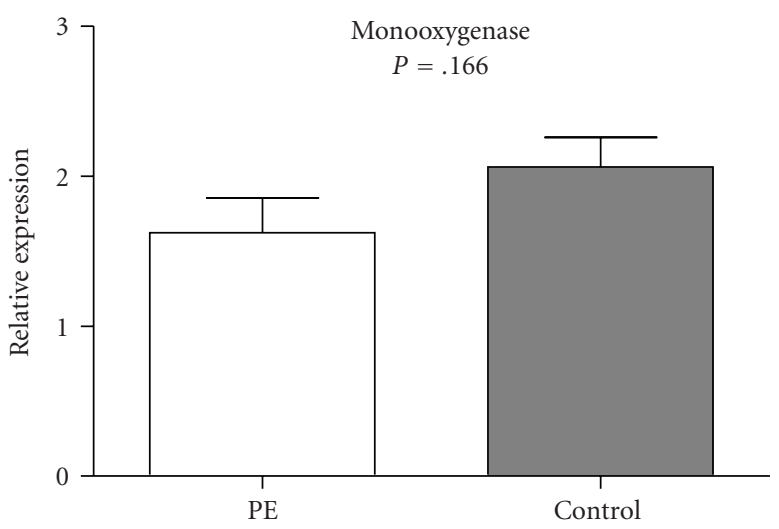

(c)

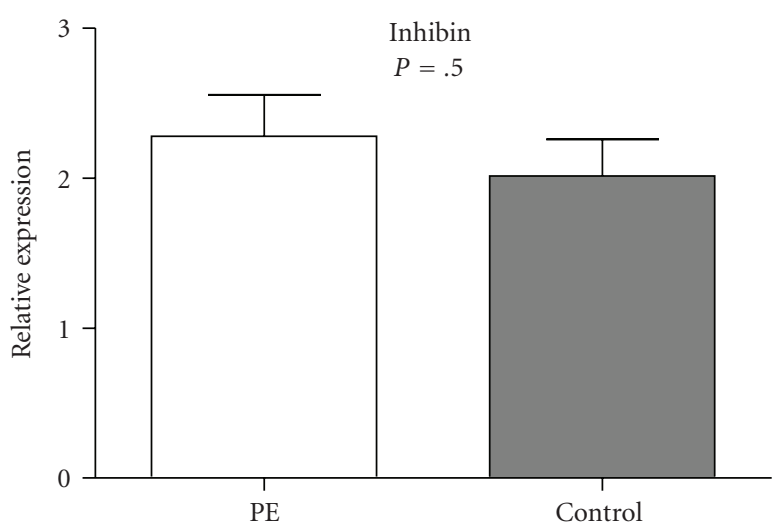

(b)

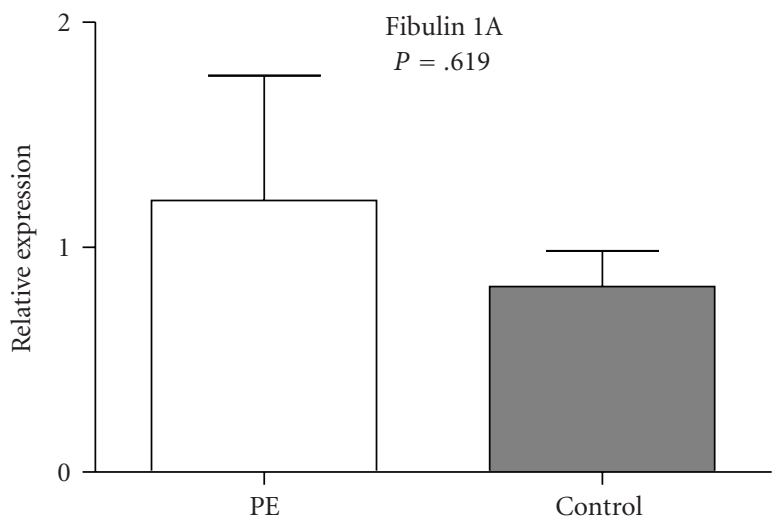

(d)

FIgURE 2: The expression patterns of the sequences as determined by real-time PCR. White bars represent pre-eclampsia cases; grey bars represent controls. Each bar represents the mean (+SEM) relative expression of the individual samples in the experiment. $P$ values for comparisons between cases and controls were: Fibulin (.619; FC 1.46), leptin (.012; FC 3.41), inhibin (.500; FC 1.13), tyr 3monooxygenase/tryp 5-monooxygenase activation protein $\varepsilon(P=.166$; FC -1.27$)$; Mann-Whitney test. (Pre-eclampsia cases: $n=9$, controls: $n=12$; one control placenta was found to have a cDNA yield which was significantly lower than the other samples and it was therefore excluded from the subsequent analyses).

to the rise in serum concentrations, possibly more so for activin A than for inhibin A [22]. Activin A is also involved in inflammatory responses, though it seems to be dependent on dose whether the effect it exerts is anti- or proinflammatory [23]. Thus activin A may play a role in the maternal inflammatory response observed in pre-eclampsia. Microarray analysis indicated a 2.06-fold upregulation of inhibin beta A subunit, which was confirmed by real-time PCR (1.9-fold up-regulation). There is an increase of activin A levels in pre-eclampsia. Inhibin beta A subunit is part of activin A and may therefore directly contribute to the increased levels observed.

4.1.5. Hypertension. The eicosanoids such as prostaglandin and thromboxane are important mediators of blood vessel contractility and blood pressure regulation. We found two enzymes involved in the eicosanoid metabolism to be regulated between the pre-eclamptic placentas and the normal controls-hydroxyprostaglandin dehydrogenase 15 and prostaglandin D2 synthase. Hydroxyprostaglandin dehydrogenase 15 (15-PGDH, type I) is the main enzyme converting prostaglandins E2 and F2 to their biologically inactive 15keto derivatives. Prostaglandin acts as a vasodilator. One study has found a twofold reduction of PGDH mRNA levels in placental tissue of patients with pre-eclampsia, compared with matched controls [24]. Others have found an increase in the activity of PGHD in pre-eclamptic placentas [25]. We found an upregulation (1.48-fold) of PGDH in the preeclamptic placentas. PGDH is involved in parturition and the expression is therefore dependent of the mode of delivery. During normal pregnancy at full term, PGDH transcription and activity in the chorio-decidua is reduced [26], allowing prostaglandins to reach the myometrium and to increase myometrial contractility. The placental expression does not seem to be affected [26]. In our study, a majority of the women in the PE group delivered vaginally, while almost all of the controls underwent elective Caesarean sections. Even taking this bias into account, the effect of parturition in the PE group should not be an increase of placental PGDH and it is therefore possible that PGDH plays a role in the pathogenesis of PE. Increased levels of PGDH would lead to decreased levels of biologically active vasodilatory 
prostaglandins, which might contribute to a hypertensive state. Since results from different studies on the placental PGDH expression in PE are conflicting, further investigation is necessary.

Glutathione- (GSH-) independent PGD2 synthase catalyzes the conversion of prostaglandin H2 (PGH2) to PGD2 in the presence of various sulfhydryl compounds. One study showed that PGD2 synthase concentration in amniotic fluid is decreased in abnormal pregnancies [27]. It has been suggested that PGD2 might contribute to the maintenance of pregnancy by controlling the Th1/Th2 balance and antigen presentation by dendritic cells through a dual receptor system [28]. Prostaglandins are vasodilators and a downregulation of prostaglandin D2 synthase as identified by our microarray analysis (1.51-fold) may contribute to the hypertensive state of PE. Its aberrant expression may also influence the reversal of the Th1/Th2 balance from that of normal pregnancies to that of pre-eclampsia.

Hypoxia-inducible protein 2 (hypoxia-inducible factor 2 alpha (HIF2A)) is a transcription factor, which is activated by hypoxic conditions. It is capable of specifically activating the transcription of the endothelial tyrosine kinase gene Tie-2 involved in angiogenesis [29]. Knock-out experiments in mice have shown that HIF2A also plays a role in the up-regulation of expression of the vasoconstrictors catecholamine and endothelin-1 $[30,31]$. We found HIF2A mRNA expression up-regulated by 1.61 -fold in PE. Others have previously found HIF2A protein, but not mRNA, expression upregulated in pre-eclamptic placentas [32]. The same investigators also found that villous explants from PE placentas fail to adequately downregulate hypoxia-induced HIF protein expression upon oxygenation [33].

Bradykinin B1 receptor is an inducible receptor, expressed during inflammatory conditions under the influence of cytokines. It mediates a vasorelaxing effect by inducing nitric oxide release. Bradykinin is a powerful angiogenic stimulus in vivo and in vitro. We found the expression of bradykinin receptor $\mathrm{B} 1$ in pre-eclamptic placentas to be 1.44 fold less than that in the normal controls, indicating that it might be involved in the development of hypertension and insufficient placental vascular growth associated with $\mathrm{PE}$.

The leptin hormone is produced by adipose tissue and by the placenta during pregnancy [34]. In nonpregnant humans, leptin negatively regulates food intake and body weight via its receptor in the hypothalamus [34]. Leptin has also been shown to correlate with plasma insulin levels and an association between insulin resistance and resistance to leptin has been suggested [35]. In normal pregnancy, leptin levels increase, particularly in the second trimester, and decline postpartum [36]. Several studies have found leptin even further upregulated in pre-eclamptic pregnancies (see review by Poston [37]) while others have not been able to prove this association [38, 39] and one study has found that leptin levels were decreased in pre-eclampsia [40]. Some have suggested that upregulation of leptin in preeclampsia may cause maternal leptin resistance, which may be a component of insulin resistance, predisposing the onset of endothelial dysfunction [35]. An increase in maternal leptin levels may cause a rise in blood pressure through stimulation of sympathic activity, or it may have beneficial consequences, such as stimulation of fetal growth. It has been proposed that leptin controls the functional integrity of the feto-placental unit thereby maintaining pregnancy by virtue of its immunoregulatory property via T lymphocytes or proto-oncogenes [41]. We found leptin to be upregulated in the placenta in pre-eclamptic pregnancies. Others have also found leptin levels upregulated by microarray analysis $[42,43]$. Our microarray analysis indicated an upregulation of 3.2-fold. Real-time PCR analysis yielded an up-regulation of approximately 32-fold. It shall be noted that such large differences in fold-change between microarray and corresponding PCR experiments are often observed.

4.1.6. Miscellaneous. A number of hypothetical proteins and gene sequences with unknown functions were also found to have expression levels that were markedly different in the pre-eclamptic group compared to the control group. Further investigation is needed before their relevance to the development of PE can be elucidated.

4.2. Microarray Analysis. The real-time PCR experiments in general exhibited the same trends of regulation that were evident from the array analyses. The risk of single samples biasing the overall results will always be a downside to analysis of pooled materials on microarrays and the importance of additional experiments to confirm the microarray results must be emphasized. Microarray studies of placental gene expression in pre-eclamptic placentas versus a control group data analysis with a specified set of parameters showed 59 genes [42], 96 genes [44], 137 genes [45], and 36 genes $[45,46]$ significantly differentially regulated between the two groups. Three of the genes exhibiting up-regulation are also present among the up-regulated genes identified in our study, namely, collagen XVII alpha 1, leptin, and inhibin beta A subunit. When comparing results from different casecontrol gene expression studies-microarrays, proteomic studies, or even gene-specific studies, several aspects must be taken into consideration. Firstly, differences in detection limits and analysis parameters may exist. Secondly, the design of the case and control groups will influence the results. The clinical characteristics, such as mean gestational age and parity of the included patients, will be of importance as well as the cellular composition of the placental samples. The mean gestational age of our study is higher (37.9 weeks for the samples studied on the microarrays) than that of the study of Reimer et al. [42] (mean gestational age 30.7 weeks) and unlike Reimer et al. we did not remove maternal decidual tissue from the placental biopsies. Our control group consists of women that have had uncomplicated pregnancies until delivery, the main part of which delivered by elective Caesarean section due to indications unrelated to the course of pregnancy. Finally, our study utilized the U133A chip, which represents sequences from $\sim 15,000$ genes.

The previous studies of gene expression in pre-eclamptic and control placental samples have revealed differences in the expression of immunologic factors. This was also observed in the microarray study by Reimer et al. [42]. For example, 
Human Leukocyte Antigen-G (HLA-G) has been found to be differentially expressed in several studies. By relaxing the parameters of our data analysis (specifically by filtering the genes according to the upper bound value of the confidence interval for the fold changes), immunological genes such as T-cell receptor gamma-delta, killer cell immunoglobulin-like receptors, and HLA-G were also found to exhibit differences in expression between pre-eclampsia pools and controls. In some of the previously published studies, gestational ages were also shorter and other genes may be important in the pathogenesis of pre-eclampsia at these stages of pregnancy. So even though this study did not identify immunological factors as being differentially expressed, an immunological component in the pathogenesis of pre-eclampsia cannot be excluded for the first and second trimesters of pregnancy.

In conclusion, microarray analysis of the gene expression in pre-eclamptic placentas compared to that of normal controls indicated the regulation of a surprisingly small number of genes. However, the fact that others have found some of these genes regulated in connection with pre-eclampsia strengthens the credibility of the results of this study. Leptin and inhibin beta A subunit were both found to be up-regulated, which was confirmed by realtime PCR and which is consistent with the findings in other studies. These and other secreted factors may be of importance to the development of the maternal vascular endothelial dysfunction observed in pre-eclampsia. Apart from placental secreted proteins, other placental factors, perhaps including 14-3-3 proteins, working to increase the shedding of syncytiotrophoblast microvillous membranes (STBMs) into the maternal circulation may also contribute to this, since STBMs are found at higher concentration in the circulation of pre-eclamptic women compared to women with uncomplicated pregnancies [18]. Genes previously found to be connected with $\mathrm{PE}$, such as thioredoxin reductase, hydroxyprostaglandin dehydrogenase, and hypoxia-inducible protein 2 alpha, were also identified by our array analysis. Furthermore, the microarray analysis yielded differential expression patterns between cases and controls for a number of novel factors not previously connected with PE, for example, prostaglandin D2 synthase and TRAP240. Surprisingly, no immunological factors were identified, but this may be because the pathways that we find regulated in this study represent the endpoint of what must be presumed to be a cascade of events effectuated throughout gestation. Early immune maladaptation may contribute to the shallow trophoblast invasion of the spiral arteries and the lack of spiral artery conversion into highcapacitance vessels observed in PE. This leads to a reduced placental perfusion and therefore a reduced nutrient supply and the generation of a hypoxic placental environment. Some of the genes identified in this study are hypoxia inducible transcription factors. Other genes implicated in gene transcription, such as additional transcription factors and proteins involved in growth factors signalling, were also found to be regulated. We hypothesize that factors induced by hypoxia or a reduced nutrient supply may contribute to an alteration of the regulation of gene expression in the pre-eclamptic placenta. Thus, some of the genes previously reported to be differentially expressed in pre-eclampsia may be so due to an aberrant regulation of expression rather than dysfunctional genes themselves. This, of course, does not mean that certain polymorphisms or mutations may not predispose to the development of PE and indeed several studies have shown associations between $\mathrm{PE}$ and genetic polymorphisms. These observations may partly explain the complexity of the disease and the vast number of genes [8] that have been implicated in the pathogenesis of preeclampsia up till now.

\section{Acknowledgments}

The authors would like to thank laboratory technician Susanne Smed for her assistance with the microarray experiments and bioinformatician Margrethe Schang Rasmussen for her assistance with the dChip software. This work was supported by grants from the Pharmacy Foundation of 1991, the A.P. Møller Foundation for the Advancement of Medical Science, the Danish Medical Association Research Fund, and H:S Research Fund.

\section{References}

[1] "Geographic variation in the incidence of hypertension in pregnancy. World Health Organization International Collaborative Study of Hypertensive Disorders of Pregnancy," American Journal of Obstetrics \& Gynecology, vol. 158, pp. 8083, 1988.

[2] E. van Beek and L. L. H. Peeters, "Pathogenesis of preeclampsia: a comprehensive model," Obstetrical and Gynecological Survey, vol. 53, no. 4, pp. 233-239, 1998.

[3] L. Duley, "The global impact of pre-eclampsia and eclampsia," Seminars in Perinatology, vol. 33, no. 3, pp. 130-137, 2009.

[4] F. Lyall, "The human placental bed revisited," Placenta, vol. 23, no. 8-9, pp. 555-562, 2002.

[5] F. Lyall, "Development of the utero-placental circulation: the role of carbon monoxide and nitric oxide in trophoblast invasion and spiral artery transformation," Microscopy Research and Technique, vol. 60, no. 4, pp. 402-411, 2003.

[6] M. J. VanWijk, K. Kublickiene, K. Boer, and E. VanBavel, "Vascular function in preeclampsia," Cardiovascular Research, vol. 47, no. 1, pp. 38-48, 2000.

[7] T. Kajii and K. Ohama, "Androgenetic origin of hydatidiform mole," Nature, vol. 268, no. 5621, pp. 633-634, 1977.

[8] A. M. Lachmeijer, G. A. Dekker, G. Pals, J. G. Aarnoudse, L. P. ten Kate, and R. Arngrímsson, "Searching for preeclampsia genes: the current position," European Journal of Obstetrics Gynecology and Reproductive Biology, vol. 105, no. 2, pp. 94113, 2002.

[9] R. T. Lie, S. Rasmussen, H. Brunborg, H. K. Gjessing, E. LieNielsen, and L. M. Irgens, "Fetal and maternal contributions to risk of pre-eclampsia: population based study," British Medical Journal, vol. 316, no. 7141, pp. 1343-1347, 1998.

[10] C. Li and W. H. Wong, "Model-based analysis of oligonucleotide arrays: expression index computation and outlier detection," Proceedings of the National Academy of Sciences of the United States of America, vol. 98, no. 1, pp. 31-36, 2001.

[11] J. D. Fondell, H. Ge, and R. G. Roeder, "Ligand induction of a transcriptionally active thyroid hormone receptor coactivator complex," Proceedings of the National Academy of Sciences of 
the United States of America, vol. 93, no. 16, pp. 8329-8333, 1996.

[12] T. Maruo, H. Matsuo, and M. Mochizuki, "Thyroid hormone as a biological amplifier of differentiated trophoblast function in early pregnancy," Acta Endocrinologica, vol. 125, no. 1, pp. 58-66, 1991.

[13] H. Matsuo, T. Maruo, K. Murata, and M. Mochizuki, "Human early placental trophoblasts produce an epidermal growth factor-like substance in synergy with thyroid hormone," Acta Endocrinologica, vol. 128, no. 3, pp. 225-229, 1993.

[14] R. Kumar and B. N. Chaudhuri, "Altered maternal thyroid function: fetal and neonatal development of rat," Indian Journal of Physiology and Pharmacology, vol. 33, no. 4, pp. 233238, 1989.

[15] E. Shibata, K. Ejima, H. Nanri, et al., "Enhanced protein levels of protein thiol/disulphide oxidoreductases in placentae from pre-eclamptic subjects," Placenta, vol. 22, no. 6, pp. 566-572, 2001.

[16] K. Ejima, T. Koji, H. Nanri, M. Kashimura, and M. Ikeda, "Expression of thioredoxin and thioredoxin reductase in placentae of pregnant mice exposed to lipopolysaccharide," Placenta, vol. 20, no. 7, pp. 561-566, 1999.

[17] M. Rosenquist, "14-3-3 proteins in apoptosis," Brazilian Journal of Medical and Biological Research, vol. 36, no. 4, pp. 403-408, 2003.

[18] M. Knight, C. W. G. Redman, E. A. Linton, and I. L. Sargent, "Shedding of syncytiotrophoblast microvilli into the maternal circulation in pre-eclamptic pregnancies," British Journal of Obstetrics and Gynaecology, vol. 105, no. 6, pp. 632-640, 1998.

[19] N. A. Bersinger, A. K. Smarason, S. Muttukrishna, N. P. Groome, and C. W. Redman, "Women with preeclampsia have increased serum levels of pregnancy-associated plasma protein A (PAPP-A), inhibin A, activin A, and soluble E-selectin," Hypertension in Pregnancy, vol. 22, no. 1, pp. 45-55, 2003.

[20] P. Florio, P. Ciarmela, S. Luisi, et al., "Pre-eclampsia with fetal growth restriction: placental and serum activin A and inhibin A levels," Gynecological Endocrinology, vol. 16, no. 5, pp. 365372, 2002.

[21] U. Manuelpillai, M. Schneider-Kolsky, P. Thirunavukarasu, A. Dole, K. Waldron, and E. M. Wallace, "Effect of hypoxia on placental activin A, inhibin A and follistatin synthesis," Placenta, vol. 24, no. 1, pp. 77-83, 2003.

[22] H. M. Silver, G. M. Lambert-Messerlian, F. M. Reis, A. M. Diblasio, F. Petraglia, and J. A. Canick, "Mechanism of increased maternal serum total activin A and inhibin A in preeclampsia," Journal of the Society for Gynecologic Investigation, vol. 9, no. 5, pp. 308-312, 2002.

[23] D. J. Phillips, K. L. Jones, J.-P. Y. Scheerlinck, M. P. Hedger, and D. M. de Kretser, "Evidence for activin A and follistatin involvement in the systemic inflammatory response," Molecular and Cellular Endocrinology, vol. 180, no. 1-2, pp. 155-162, 2001.

[24] E. Schoof, M. Girstl, W. Frobenius, et al., "Decreased gene expression of $11 \beta$-hydroxysteroid dehydrogenase type 2 and 15-hydroxyprostaglandin dehydrogenase in human placenta of patients with preeclampsia," Journal of Clinical Endocrinology and Metabolism, vol. 86, no. 3, pp. 1313-1317, 2001.

[25] J. Jarabak, J. D. Watkins, and M. Lindheimer, "In vitro activity of nicotinamide adenine dinucleotide- and nicotinamide adenine dinucleotide phosphate-linked 15 hydroxyprostaglandin dehydrogenases in placentas from normotensive and preeclamptic/eclamptic pregnancies," Journal of Clinical Investigation, vol. 80, no. 4, pp. 936-940, 1987.
[26] R. K. Sangha, J. C. Walton, C. M. Ensor, H.-H. Tai, and J. R. G. Challis, "Immunohistochemical localization, messenger ribonucleic acid abundance, and activity of 15hydroxyprostaglandin dehydrogenase in placenta and fetal membranes during term and preterm labor," Journal of Clinical Endocrinology and Metabolism, vol. 78, no. 4, pp. 982989, 1994.

[27] D. N. Melegos, H. Yu, and E. P. Diamandis, "Prostaglandin D2 synthase: a component of human amniotic fluid and its association with fetal abnormalities," Clinical Chemistry, vol. 42, no. 7, pp. 1042-1050, 1996.

[28] S. Saito, H. Tsuda, and T. Michimata, "Prostaglandin D2 and reproduction," American Journal of Reproductive Immunology, vol. 47, no. 5, pp. 295-302, 2002.

[29] H. Tian, S. L. McKnight, and D. W. Russell, "Endothelial PAS domain protein 1 (EPAS1), a transcription factor selectively expressed in endothelial cells," Genes and Development, vol. 11, no. 1, pp. 72-82, 1997.

[30] H. Tian, R. E. Hammer, A. M. Matsumoto, D. W. Russell, and S. L. McKnight, "The hypoxia-responsive transcription factor EPAS1 is essential for catecholamine homeostasis and protection against heart failure during embryonic development," Genes and Development, vol. 12, no. 21, pp. 3320-3324, 1998.

[31] K. Brusselmans, V. Compernolle, M. Tjwa, et al., "Heterozygous deficiency of hypoxia-inducible factor- $2 \alpha$ protects mice against pulmonary hypertension and right ventricular dysfunction during prolonged hypoxia," Journal of Clinical Investigation, vol. 111, no. 10, pp. 1519-1527, 2003.

[32] A. Rajakumar, K. A. Whitelock, L. A. Weissfeld, A. R. Daftary, N. Markovic, and K. P. Conrad, "Selective overexpression of the hypoxia-inducible transcription factor, HIF- $2 \alpha$, in placentas from women with preeclampsia," Biology of Reproduction, vol. 64, no. 2, pp. 499-506, 2001.

[33] A. Rajakumar, K. Doty, A. Daftary, G. Harger, and K. P. Conrad, "Impaired oxygen-dependent reduction of HIF-1 $\alpha$ and $-2 \alpha$ proteins in pre-eclamptic placentae," Placenta, vol. 24, no. 2-3, pp. 199-208, 2003.

[34] N. Sagawa, S. Yura, H. Itoh, et al., "Possible role of placental leptin in pregnancy: a review," Endocrine, vol. 19, no. 1, pp. 65-71, 2002.

[35] C. M. Anderson and J. Ren, "Leptin, leptin resistance and endothelial dysfunction in pre-eclampsia," Cellular and Molecular Biology (Noisy-le-Grand), vol. 48, pp. OL323-OL329, 2002.

[36] E. Domali and I. E. Messinis, "Leptin in pregnancy," Journal of Maternal-Fetal and Neonatal Medicine, vol. 12, no. 4, pp. 222230, 2002.

[37] L. Poston, "Leptin and preeclampsia," Seminars in Reproductive Medicine, vol. 20, no. 2, pp. 131-138, 2002.

[38] N. A. Bersinger, N. Groome, and S. Muttukrishna, "Pregnancy-associated and placental proteins in the placental tissue of normal pregnant women and patients with preeclampsia at term," European Journal of Endocrinology, vol. 147, no. 6, pp. 785-793, 2002.

[39] E. Martinez-Abundis, M. Gonzalez-Ortiz, and S. PascoeGonzalez, "Serum leptin levels the severity of preeclampsia," Archives of Gynecology and Obstetrics, vol. 264, no. 2, pp. 71$73,2000$.

[40] M. C. Henson and V. D. Castracane, "Leptin: roles and regulation in primate pregnancy," Seminars in Reproductive Medicine, vol. 20, no. 2, pp. 113-121, 2002.

[41] R. Bajoria, S. R. Sooranna, B. S. Ward, and R. Chatterjee, "Prospective function of placental leptin at maternal-fetal interface," Placenta, vol. 23, no. 2-3, pp. 103-115, 2002. 
[42] T. Reimer, D. Koczan, B. Gerber, D. Richter, H. J. Thiesen, and K. Friese, "Microarray analysis of differentially expressed genes in placental tissue of pre-eclampsia: up-regulation of obesityrelated genes," Molecular Human Reproduction, vol. 8, no. 7, pp. 674-680, 2002.

[43] D. A. Enquobahrie, M. Meller, K. Rice, B. M. Psaty, D. S. Siscovick, and M. A. Williams, "Differential placental gene expression in preeclampsia," American Journal of Obstetrics and Gynecology, vol. 199, no. 5, pp. 566.e1-566.e11, 2008.

[44] R. Zhou, Q. Zhu, Y. Wang, Y. Ren, L. Zhang, and Y. Zhou, "Genomewide oligonucleotide microarray analysis on placentae of pre-eclamptic pregnancies," Gynecologic and Obstetric Investigation, vol. 62, no. 2, pp. 108-114, 2006.

[45] H. Nishizawa, K. Pryor-Koishi, T. Kato, H. Kowa, H. Kurahashi, and Y. Udagawa, "Microarray analysis of differentially expressed fetal genes in placental tissue derived from early and late onset severe pre-eclampsia," Placenta, vol. 28, no. 5-6, pp. 487-497, 2007.

[46] S. A. Founds, J. S. Dorman, and Y. P. Conley, "Microarray technology applied to the complex disorder of Preeclampsia," Journal of Obstetric, Gynecologic, and Neonatal Nursing, vol. 37, no. 2, pp. 146-157, 2008. 

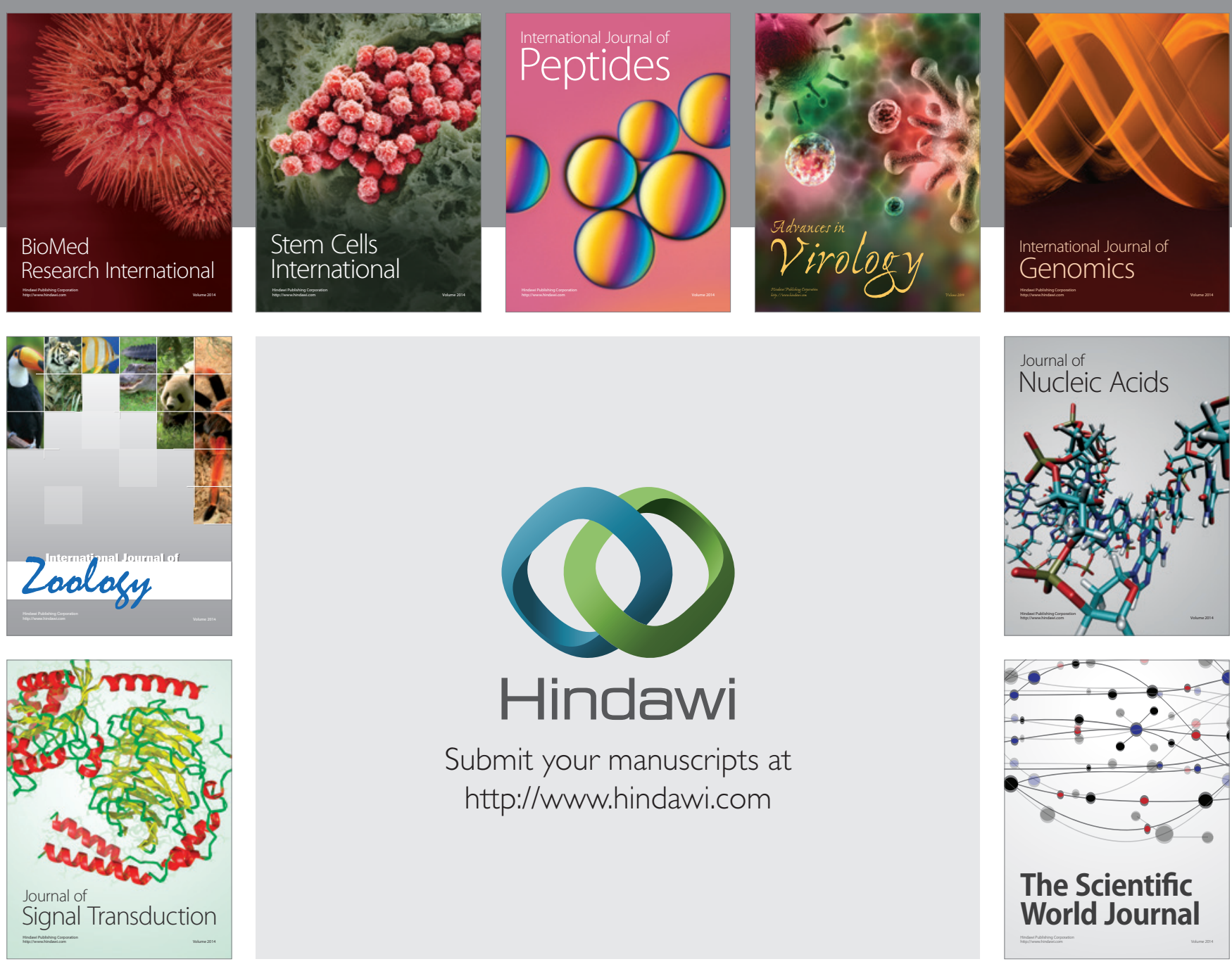

Submit your manuscripts at

http://www.hindawi.com
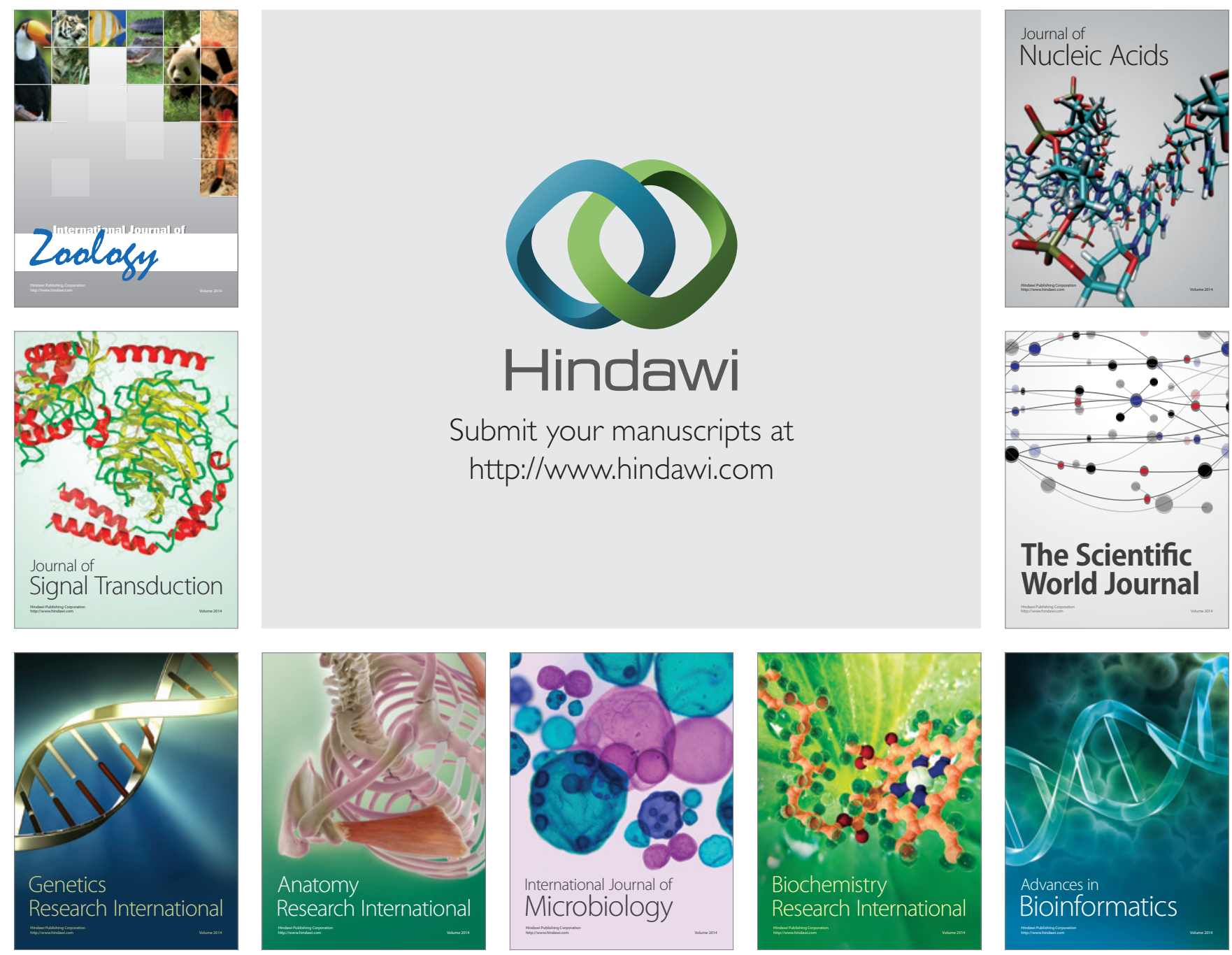

The Scientific World Journal
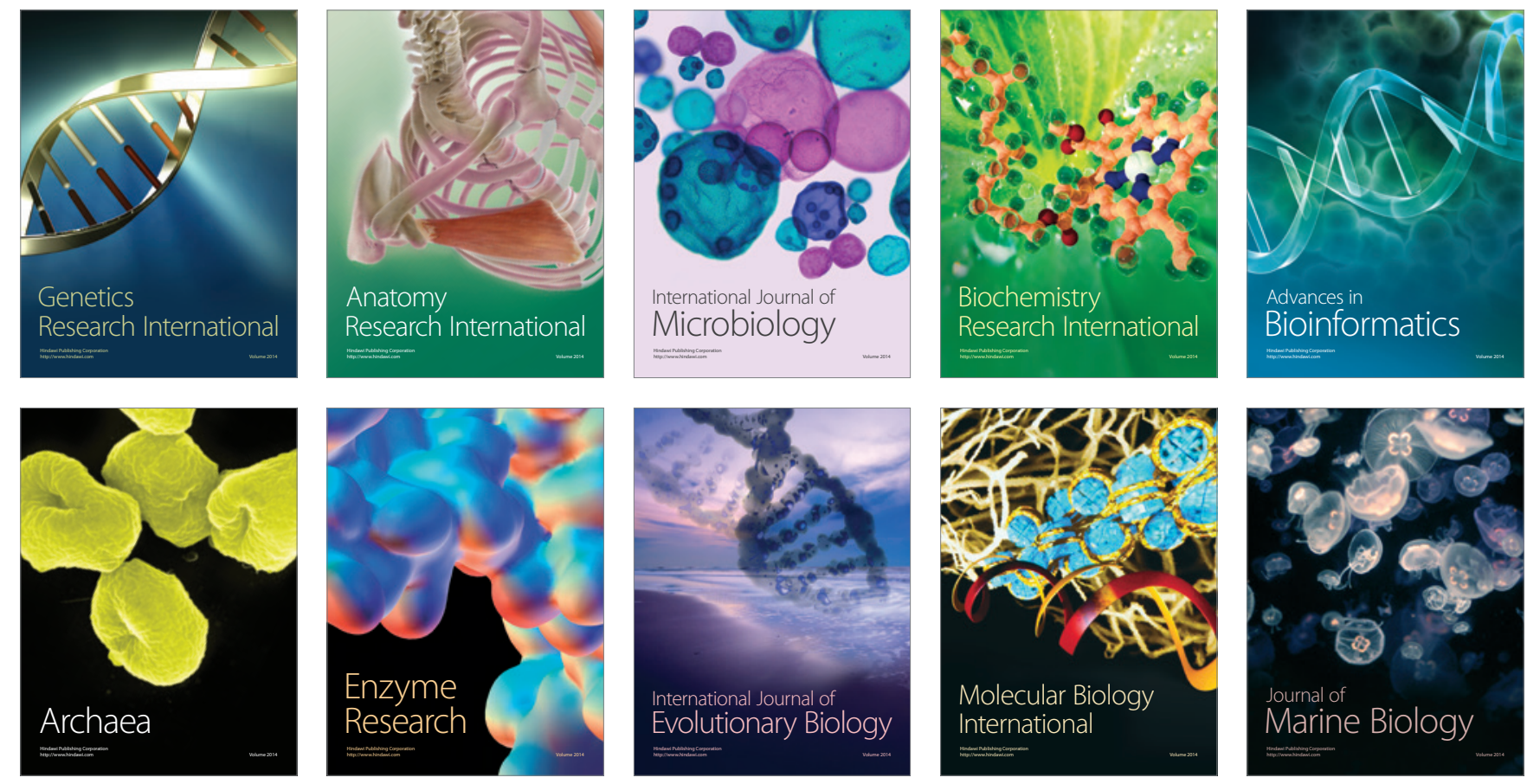